\title{
Comparisons of genetic population structures in four intertidal brachyuran species of contrasting habitat characteristics
}

\author{
Masako Kawane $\cdot$ Keiji Wada $\cdot$ Katsutoshi Watanabe
}

Published online: 28 November 2008

(C) Springer-Verlag 2008

Erratum to: Mar Biol (2008) 156:193-203

DOI 10.1007/s00227-008-1076-y

Unfortunately, the presentation of Tables $1,2,3$, and 4 contain errors. The correct tables are given below.

The online version of the original article can be found under doi:10.1007/s00227-008-1076-y.

M. Kawane $\cdot$ K. Wada $(\bowtie)$

Department of Biological Science, Faculty of Science,

Nara Women's University, Kitauoya-nishimachi,

Nara 630-8506, Japan

e-mail: mbanzai@cc.nara-wu.ac.jp

K. Watanabe

Laboratory of Animal Ecology, Graduate School of Science,

Kyoto University, Kitashirakawa-Oiwakecho,

Sakyo-ku, Kyoto 606-8502, Japan 
Table 1 Haplotype composition, haplotype diversity and nucleotide diversity of 10 Ocypode ceratophthalma population

\begin{tabular}{|c|c|c|c|c|c|c|c|c|c|c|c|c|c|c|c|c|}
\hline \multirow{2}{*}{$\begin{array}{l}\text { Localty } \\
\text { number }\end{array}$} & \multicolumn{16}{|c|}{ Haplotype } \\
\hline & $\mathrm{Oc} 1$ & Oc2 & Oc3 & Oc4 & Oc5 & Oc6 & Oc7 & Oc8 & Oc9 & Oc10 & \multicolumn{2}{|c|}{ Oc11 } & Oc12 & Oc13 & Oc14 & Oc15 \\
\hline 6 & 4 & 1 & 2 & 1 & 2 & & & & & & & & & & & \\
\hline 9 & 2 & 1 & & 4 & 1 & 1 & 1 & & & & & & & & & \\
\hline 16 & 4 & & 2 & 1 & 1 & & & 1 & 1 & & & & & & & \\
\hline 18 & 3 & & & 2 & 3 & & & & & 1 & 1 & & & & & \\
\hline 23 & 2 & & & 1 & 1 & & & & & & & & 1 & 1 & 1 & 1 \\
\hline 27 & 1 & & & 2 & 1 & & & & & & & & & & & \\
\hline 32 & 3 & & & 2 & 3 & & & & & & & & & & & \\
\hline 33 & 2 & & 1 & 4 & & & & & & & & & & & & \\
\hline 34 & 1 & & & 1 & & & & & & & & & & & & \\
\hline 35 & 1 & & & & & & & & & & & & & & & \\
\hline Total & 23 & 2 & 5 & 18 & 12 & 1 & 1 & 1 & 1 & 1 & 1 & & 1 & 1 & 1 & 1 \\
\hline \multirow{2}{*}{$\begin{array}{l}\text { Localty } \\
\text { number }\end{array}$} & \multicolumn{11}{|c|}{ Haplotype } & \multirow[t]{2}{*}{$n$} & \multirow{2}{*}{\multicolumn{2}{|c|}{$\begin{array}{l}\text { Haplotype } \\
\text { diversity }\end{array}$}} & \multirow{2}{*}{\multicolumn{2}{|c|}{$\begin{array}{l}\text { Nucleotide } \\
\text { diversity }\end{array}$}} \\
\hline & Oc16 & Oc17 & Oc18 & Oc19 & Oc20 & Oc21 & Oc22 & Oc23 & Oc24 & Oc25 & Oc26 & & & & & \\
\hline 6 & & & & & & & & & & & & 10 & 0.822 & $=0.097$ & 0.0022 & 0.0018 \\
\hline 9 & & & & & & & & & & & & 10 & 0.844 & $=0.103$ & 0.0035 & 0.0025 \\
\hline 16 & & & & & & & & & & & & 10 & 0.844 & $=0.103$ & 0.0085 & 0.0052 \\
\hline 18 & & & & & & & & & & & & 10 & 0.844 & $=0.080$ & 0.0033 & 0.0024 \\
\hline 23 & 1 & 1 & & & & & & & & & & 10 & 0.978 & $=0.054$ & 0.0043 & 0.0029 \\
\hline 27 & & & 1 & & & & & & & & & 5 & 0.900 & 0.161 & 0.0028 & 0.0024 \\
\hline 32 & & & & 1 & 1 & & & & & & & 10 & 0.844 & $=0.080$ & 0.0026 & 0.0020 \\
\hline 33 & 1 & & 1 & & & 1 & & & & & & 10 & 0.844 & $=0.103$ & 0.0031 & 0.0023 \\
\hline 34 & & & & & & & 1 & 1 & 1 & & & 5 & 1.000 & $=0.127$ & 0.0053 & 0.0039 \\
\hline 35 & & & & & & & & & & 1 & 3 & 5 & 0.700 & $=0.218$ & 0.0203 & 0.0131 \\
\hline Total & 2 & 1 & 2 & 1 & 1 & 1 & 1 & 1 & 1 & 1 & 3 & 85 & 0.862 & $=0.084$ & 0.0056 & 0.0055 \\
\hline
\end{tabular}

Locality numbers as given in Fig. 1 
Table 2 Haplotype composition, haplotype diversity and nucleotide diversity of 10 Gaetice depressus population

\begin{tabular}{|c|c|c|c|c|c|c|c|c|c|c|c|c|c|c|c|c|c|}
\hline \multirow{2}{*}{$\begin{array}{l}\text { Localty } \\
\text { number }\end{array}$} & \multicolumn{17}{|c|}{ Haplotype } \\
\hline & $\mathrm{Gd} 1$ & $\mathrm{Gd} 2$ & $\mathrm{Gd} 3$ & \multicolumn{2}{|c|}{$\mathrm{Gd} 4$} & Gd5 & Gd6 & $\mathrm{Gd} 7$ & Gd8 & Gd9 & Gd10 & \multicolumn{2}{|l|}{ Gd11 } & Gd12 & Gd13 & Gd14 & Gd15 \\
\hline 1 & 5 & 1 & 3 & 1 & 1 & & 1 & & & & & & & & & & \\
\hline 3 & 3 & & 3 & & & & & 1 & 1 & 1 & 1 & & & & & & \\
\hline 8 & 5 & & 2 & & & & & & & & 1 & 1 & & 1 & & & \\
\hline 14 & 4 & & 2 & & & & & & & & & & & & 1 & 1 & 1 \\
\hline 18 & 1 & & 3 & & 1 & & & & & & & & & & & & \\
\hline 22 & 1 & & 7 & & & & & & & & & & & & & & \\
\hline 28 & 4 & & 5 & & & & & & & & & & & & & & \\
\hline \multicolumn{18}{|l|}{31} \\
\hline Total & 21 & 1 & 25 & 1 & 2 & & 1 & 1 & 1 & 1 & 2 & 1 & & 1 & 1 & 1 & 1 \\
\hline \multirow{2}{*}{$\begin{array}{l}\text { Localty } \\
\text { number }\end{array}$} & \multicolumn{12}{|c|}{ Haplotype } & $n$ & \multirow{2}{*}{\multicolumn{2}{|c|}{$\begin{array}{l}\text { Haplotype } \\
\text { diversity }\end{array}$}} & \multirow{2}{*}{\multicolumn{2}{|c|}{$\begin{array}{l}\text { Nucleotide } \\
\text { diversity }\end{array}$}} \\
\hline & $\overline{\mathrm{Gd} 16}$ & Gd17 & Gd18 & Gd19 & Gd20 & $\mathrm{Gd} 21$ & Gd 22 & Gd23 & $\mathrm{Gd} 24$ & Gd25 & Gd26 & $\overline{\mathrm{Gd} 27}$ & & & & & \\
\hline 1 & & & & & & & & & & & & & 10 & 0.867 & \pm 0.085 & 0.0027 & $=0.0021$ \\
\hline 3 & & & & & & & & & & & & & 10 & 0.867 & \pm 0.085 & 0.0036 & $=0.0025$ \\
\hline 8 & & & & & & & & & & & & & 10 & 0.756 & \pm 0.130 & 0.0034 & $=0.0025$ \\
\hline 14 & 1 & & & & & & & & & & & & 10 & 0.844 & \pm 0.103 & 0.0031 & $=0.0023$ \\
\hline 18 & & 1 & 1 & 1 & 1 & 1 & & & & & & & 10 & 0.933 & \pm 0.077 & 0.0042 & $=0.0029$ \\
\hline 22 & & & & & & & 1 & 1 & & & & & 10 & 0.533 & \pm 0.180 & 0.0012 & $=0.0012$ \\
\hline 28 & & & & & & & & & 1 & & & & 10 & 0.644 & \pm 0.101 & 0.0015 & $=0.0014$ \\
\hline 31 & & & & & & & & & & 7 & 2 & 1 & 10 & 0.511 & \pm 0.164 & 0.0011 & $=0.0011$ \\
\hline Total & 1 & 1 & 1 & 1 & 1 & 1 & 1 & 1 & 1 & 7 & 2 & 1 & 80 & 0.744 & \pm 0.163 & 0.0026 & $=0.0012$ \\
\hline
\end{tabular}

Locality numbers as given in Fig. 1 
Table 3 Haplotype composition, haplotype diversity and nucleotide diversity of 10 Chiromantes dehaani population

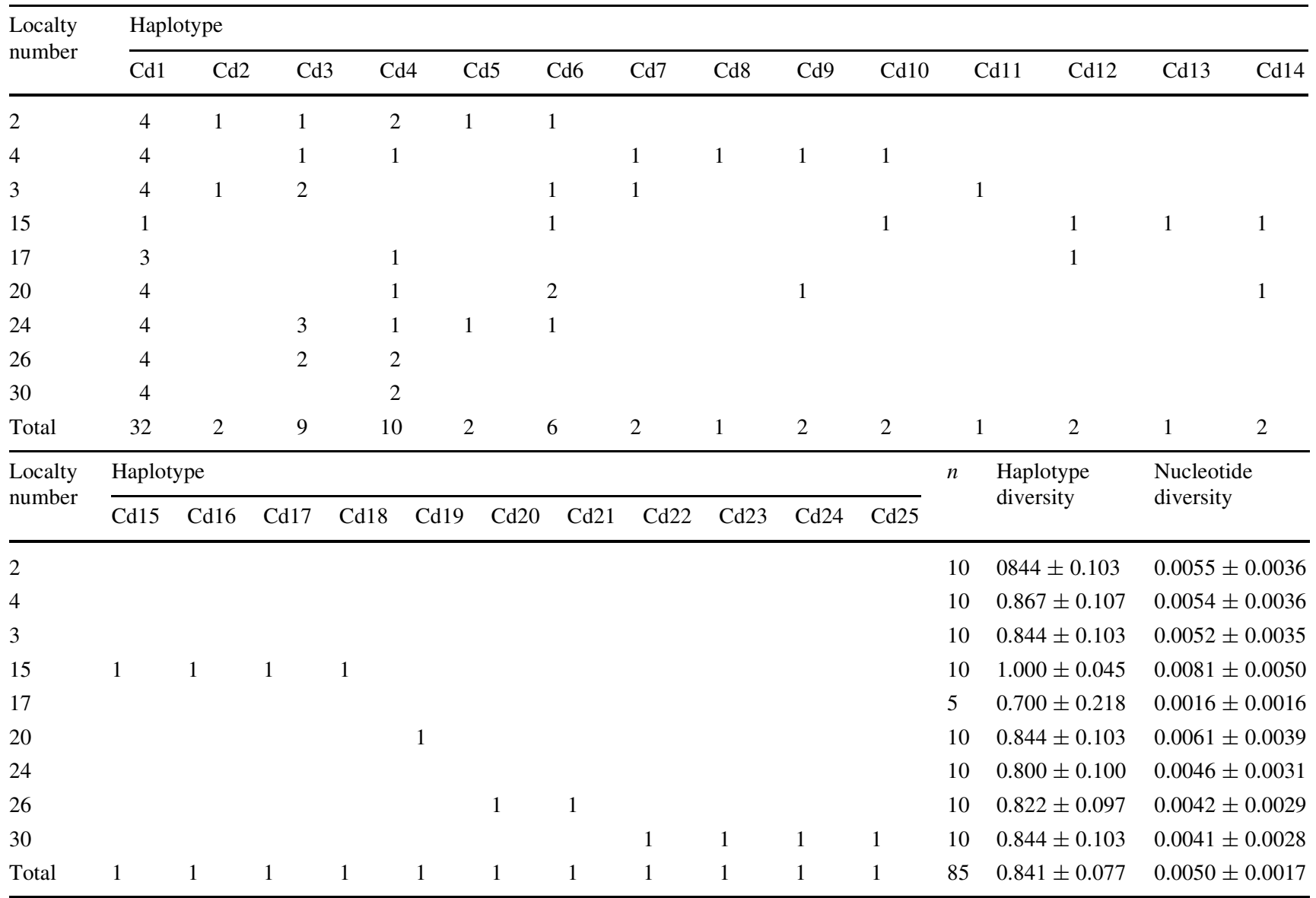

Locality numbers as given in Fig. 1 
Table 4 Haplotype composition, haplotype diversity and nucleotide diversity of 10 Deiratonotus japonicus population

\begin{tabular}{|c|c|c|c|c|c|c|c|c|c|c|c|c|c|c|c|c|c|c|c|}
\hline \multirow{2}{*}{$\begin{array}{l}\text { Localty } \\
\text { number }\end{array}$} & \multicolumn{19}{|c|}{ Haplotype } \\
\hline & $\mathrm{Dj} 1$ & $\mathrm{Dj} 2$ & $\mathrm{Dj} 3$ & $\mathrm{Dj} 4$ & Dj5 & Dj6 & Dj7 & Dj8 & Dj9 & $\mathrm{Dj} 1 \mathrm{C}$ & \multicolumn{2}{|c|}{ Dj11 } & Dj12 & Dj13 & Dj14 & Dj15 & $15 \quad \mathrm{Dj} 16 \quad \mathrm{Dj} 1$ & Dj17 & $\mathrm{Dj} 19$ \\
\hline 5 & 2 & 1 & 2 & & & & & & & & & & & & & & & & \\
\hline 14 & & & 1 & 2 & 1 & 1 & & & & & & & & & & & & & \\
\hline 11 & & & 3 & 2 & & & 1 & 1 & 1 & 1 & 1 & & & & & & & & \\
\hline 12 & & & 1 & 1 & & & & & & & & 1 & 1 & 6 & 1 & 1 & & & \\
\hline 13 & & & 4 & 3 & & & & & & & & & & 2 & & & 2 & 1 & 1 \\
\hline \multicolumn{20}{|l|}{15} \\
\hline 21 & & & 2 & & & & & & & & & & & & & & & & \\
\hline 24 & & & 1 & & & & & & & & & & & & & & & & \\
\hline \multicolumn{20}{|l|}{25} \\
\hline \multicolumn{20}{|l|}{29} \\
\hline Total & 2 & 1 & 14 & 8 & 1 & 1 & 1 & 1 & 1 & 1 & 1 & 1 & 1 & 8 & 1 & 1 & 2 & 1 & 1 \\
\hline \multirow{2}{*}{$\begin{array}{l}\text { Localty } \\
\text { number }\end{array}$} & \multicolumn{15}{|c|}{ Haplotype } & $\begin{array}{ll}n & \mathrm{H}\end{array}$ & \multirow{2}{*}{$\begin{array}{l}\text { Haplotype } \\
\text { diversity }\end{array}$} & \multirow{2}{*}{\multicolumn{2}{|c|}{$\begin{array}{l}\text { Nucleotide } \\
\text { diversity }\end{array}$}} \\
\hline & $\overline{\mathrm{Dj}} 20$ & $\mathrm{Dj} 21$ & $\mathrm{Dj} 22$ & $\mathrm{Dj} 23$ & $\mathrm{Dj} 24$ & $\mathrm{Dj} 25$ & $\mathrm{Dj} 26$ & $\mathrm{Dj} 27$ & Dj28 & Dj29 & $\mathrm{Dj} 30$ & $\mathrm{Dj} 31$ & $\mathrm{Dj} 32$ & $\mathrm{Dj} 33$ & $\overline{\mathrm{Dj} 34}$ & & & & \\
\hline 5 & & & & & & & & & & & & & & & & 50 & $0.800 \pm 0.164$ & $0.0019 \pm 0$ & 0.0017 \\
\hline 14 & & & & & & & & & & & & & & & & 50 & $0.900 \pm 0.161$ & $0.0022 \pm 0$ & 0.0020 \\
\hline 11 & & & & & & & & & & & & & & & & 100 & $0.911 \pm 0.077$ & $0.0032 \pm 0$ & 0.0023 \\
\hline 12 & & & & & & & & & & & & & & & & 110 & $0.727 \pm 0.144$ & $0.0035 \pm 0$ & 0.0024 \\
\hline 13 & 1 & & & & & & & & & & & & & & & 150 & $0.895 \pm 0.053$ & $0.0038 \pm 0$ & 0.0026 \\
\hline 15 & & 1 & 1 & 1 & 1 & 1 & & & & & & & & & & $\begin{array}{ll}5 & 1\end{array}$ & $1.000 \pm 0.127$ & $0.0041 \pm 0$ & 0.0032 \\
\hline 21 & & & & & & 1 & 1 & 1 & & & & & & & & 50 & $0.900 \pm 0.161$ & $0.0064 \pm 0$ & 0.0045 \\
\hline 24 & & & & & & & & & 1 & 1 & 1 & 1 & & & & $\begin{array}{ll}5 & 1\end{array}$ & $1.000 \pm 0.127$ & $0.0048 \pm 0$ & 0.0036 \\
\hline 25 & & & & & & & & 1 & & & & & 4 & & & 50 & $0.400 \pm 0.237$ & $0.0030 \pm 0$ & 0.0025 \\
\hline 29 & & & & & & & & & & & & & & 1 & 4 & 50 & $0.400 \pm 0.237$ & $0.0007 \pm 0$ & 0.0009 \\
\hline Total & 1 & 1 & 1 & 1 & 1 & 2 & 1 & 2 & 1 & 1 & 1 & 1 & 4 & 1 & 4 & 710 & $0.793 \pm 0.223$ & $0.0034 \pm 0$ & 0.0016 \\
\hline
\end{tabular}

Locality numbers as given in Fig. 1 